\title{
Short Exon Detection in DNA Sequences Based on Multifeature Spectral Analysis
}

\author{
Nancy Yu Song and Hong Yan \\ Department of Electronic Engineering, City University of Hong Kong, Kowloon, Hong Kong \\ Correspondence should be addressed to Nancy Yu Song, 50728680@student.cityu.edu.hk
}

Received 30 June 2010; Revised 26 August 2010; Accepted 31 October 2010

Academic Editor: Antonio Napolitano

Copyright ( $) 2011$ N. Y. Song and H. Yan. This is an open access article distributed under the Creative Commons Attribution License, which permits unrestricted use, distribution, and reproduction in any medium, provided the original work is properly cited.

\begin{abstract}
This paper presents a new technique for the detection of short exons in DNA sequences. In this method, we analyze four DNA structural properties, which include the DNA bending stiffness, disrupt energy, free energy, and propeller twist, using the autoregressive (AR) model. The linear prediction matrices for the four features are combined to find the same set of linear prediction coefficients, from which we estimate the spectrum of the DNA sequence and detect exons based on the 1/3 frequency component. To overcome the nonstationarity of DNA sequences, we use moving windows of different sizes in the AR model. Experiments on the human genome show that our multi-feature based method is superior in performance to existing exon detection algorithms.
\end{abstract}

\section{Introduction}

Signals converted from DNA sequence are nonstationary. The coding sequence of a prokaryotic gene is a contiguous series of three-nucleotide codons. The codon for one amino acid is immediately adjacent to the codon for the next amino acid in the polypeptide chain. However, this may not be the case for eukaryotic genes. Many eukaryotic genes comprise blocks of exons from each other by blocks of intons. The exons contain protein-coding instructions. Figure 1 shows a eukaryotic gene which contains three exons separated by two introns. In the transcription process, the gene sequence will firstly be transcribed into pre-mRNA. Then all the intron areas in the pre-mRNA will be spliced out and the exon areas will be joined together. This generates a mature mRNA which will be used afterwards to produce proteins [1].

The amount of genome sequence data is growing rapidly. Biological interpretations need to keep pace with the fast increase of raw sequence data. Biological experiments for gene identification in DNA sequences are costly to conduct, hence there exists a strong demand for fast and accurate computer tools to analyze the sequences, especially for finding genes and determining their functions [2]. In eukaryotic organisms, the task of gene recognition also includes distinguishing exons and introns. Moreover, this task is more complex in vertebrates than in lower eukaryotes. This is because vertebrate genes consist of multiple short exons separated by introns that are 10 or 100 times longer on average. Only $1-3 \%$ of the human genome is translated into proteins. Most of the human exons are short. The average length of human exons is $137 \mathrm{bp}$ [3].

The 3-periodicity which exists in DNA transcripts especially the protein-coding regions in a DNA sequence has been a known phenomenon for some time [4]. The periodicity is caused by uneven distribution of codons and provides a possible approach for exon identification. This paper focuses on the detection of the regions with 3-periodicity along a DNA sequence, but does not identify untranslated regions (UTRs) or nonprotein coding regions. The problem of classifying UTRs and gene expression regulatory elements in a DNA sequence has been addressed in our previous work $[5,6]$.

One direct approach of exon identification is to find splice sites. A splice sites can be recognized by some characteristic motifs. Several statistical models have been used to approximate the distributions over sets of aligned sequences, for example, based on the Markov Models and the Hidden Markov Models [7]. Another approach 


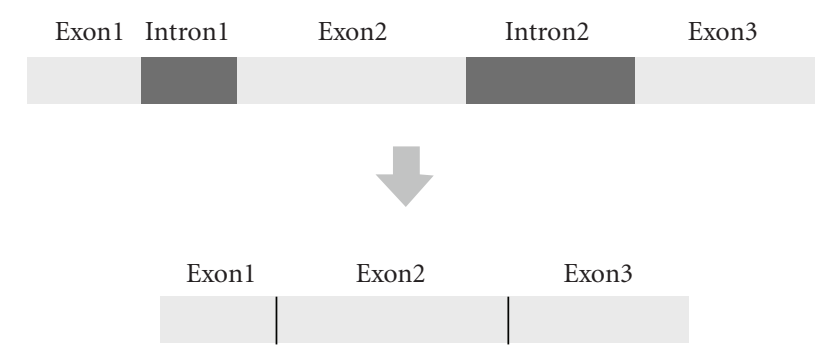

FIgURE 1: A eukaryotic gene and the splicing process.

to distinguishing exonic and intronic regions is based on digital signal processing (DSP) methods. Main DSP methods include the discrete Fourier transform, digital filters, entropy measures and spectral analysis using parametric models [8]. All these approaches look for a 3-periodic pattern in the occurrences of A, C, G or T. The Fourier transform has been widely used for sequence analysis [9]. However, the spectrum obtained by the Fourier transform contains windowing artifacts and spurious spectral peaks. Akhtar et al. proposed an optimized period-3 method which is called paired and weighted spectral rotation (PWSR) measure which takes into account both computational complexity and the relative accuracy of gene prediction [10]. Methods employing digital filters have also been developed in exon detections. Vaidyanathan and Yoon proposed a method which deploys an antinotch digital filter to find the signal energy at the $2 \pi / 3$ frequency [11]. Entropy measures are also employed in exon detection. A complexity measure based on the entropic segmentation of DNA sequences into homogeneous domains is defined by Román-Roldán et al. [12]. Nicorici and Astola proposed a method by applying recursively an entropic segmentation method on DNA sequences [13]. This method does not require prior training. Parametric models such as autoregressive modeling of DNA sequences were addressed by Chackravarthy et al. [14]. Yan and Pham proposed an AR model-based sequence analysis method to estimate the power spectral density [15]. The AR model-based analysis is able to produce stronger power spectral density peaks and weaker artifacts than the discrete Fourier transform (DFT). Choong and Yan further proposed multiscale parametric spectral analysis for exon detection based on the AR model [16]. This method is proven to be better than the DFT and previous AR modelbased methods. Jiang and Yan also used wavelet subspace Hilbert-Huang transform to identify exon regions [17]. G. Tina and T. Tessamma, proposed to denoise the signals in the coding regions using the discrete wavelet transform [18].

A problem of signal processing-based methods for finding the 3-periodicity is that it is very hard to identify short exons which are very common in human genome sequence. The 3-periodicity is essentially a very weak signal embedded in the DNA sequence and it is difficult to detect this type of signals computationally. If the exon region is short, it will be even harder to find the periodic signals.
In this paper, we propose a method to tackle the short exon identification problem based on multifeature spectral analysis. A DNA sequence is converted into numerical representations based on four DNA structural features, including the DNA-bending stiffness, disrupt energy, free energy and propeller twist. Then we perform AR model-based spectral analysis of these features to detect short exon regions. Based on experiment results, our multifeature spectral analysis method is compared with the multiscale FBLP model [16], the discrete wavelet transform denoise method [18] as well as a simple PSD addition method in this paper. The comparison shows that our method is superior in performance to the three other methods for short exon detection (Figure 2).

\section{Methodology}

2.1. Numerical Representation of a DNA Sequence. DNA is the hereditary material in humans and almost all other organisms. The structure of DNA is highly stable which makes it a perfect carrier of hereditary information. The information in DNA is stored as a code made up of four chemical bases: adenine (A), cytosine $(C)$, guanine $(G)$ and thymine (T). DNA bases pair up with each other, A with $\mathrm{T}$ and $\mathrm{C}$ with $\mathrm{G}$, forming units called base pairs. Hence a DNA sequence is naturally represented by a string which consists of "A", "C", "G" and "T". However, since DNA sequence contains a series of symbolic values, it is very hard to deal with it by signal processing methods. If the sequence could be represented by numerical values, a lot of signal processing algorithms could be applied to analyzing the sequence.

Several methods can be used to convert a DNA sequence into discrete-time signals. The most straightforward way is to assign 1 to A, 2 to $\mathrm{C}, 3$ to $\mathrm{G}$ and 4 to T. Another way is to use single-base binary representation. For a DNA sequence $[n]$, we can construct four indicator sequences as:

$$
x_{i}[n]=\left\{\begin{array}{ll}
1 & \text { if } x[n]=i \\
0 & \text { otherwise }
\end{array} \quad(i \in\{\mathrm{A}, \mathrm{C}, \mathrm{G}, \mathrm{T}\})\right.
$$

A better way is to use the double-base (DB) curve representation [19]. There are four single nucleotide bases: A, G, C, $\mathrm{T}$. The $\mathrm{DB}$ curve representation is defined as:

$$
x_{b_{1} b_{2}}(n)=\sum_{i=1}^{n} s(i), \quad n=1,2, \ldots, N
$$

where $N$ is the length of the DNA sequence and the unit numeric value $s(n)$ is defined as

$$
s(n)= \begin{cases}+1 & \text { for base } b_{1} \\ -1 & \text { for base } b_{2} \\ 0 & \text { for other bases }\end{cases}
$$




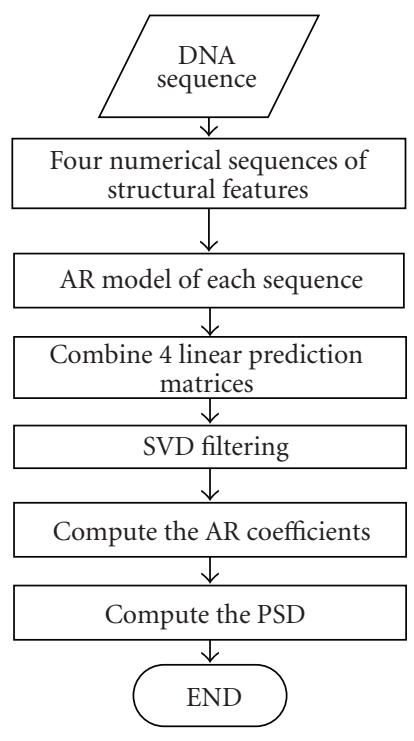

FIGURE 2: The flowchart of our algorithm for short exon detection.

where $b_{1}, b_{2} \in\{\mathrm{A}, \mathrm{G}, \mathrm{C}, \mathrm{T}\}$ and $b_{1} \neq b_{2}$. Therefore the nucleotide bases can be classified into six double-bases: AC, AG, AT, CG, CT and GT. The DB curve reflects the difference between two kinds of nucleotides along a DNA sequence. Compared to the single-base binary representation in which only the appearance of one kind of nucleotide is shown, the $\mathrm{DB}$ curve representation is much more informative. The drawback is that the number of signals to be processed increases from four to six.

All the conversion methods mentioned above are based on subjective assigned numbers. There is no biological evidence which supports the numerical assignment. DNA structural property values are obtained by physical models or biological experiments. Hence it is more reasonable to do the conversion according to DNA structural properties. Figures 3(a) and 3(b) show the PSD obtained for base pairs 6900-8100 of a DNA sequence with NCBI accession number Z20656. The actual exon positions are indicated by red rectangles. The shortest exon is only 27-bp long located at relative position 430. It is not difficult to see that there is no peak showing the existence of the 27bp long exon in Figure 3(a) which is obtained from the indicator sequences while there is an obvious peak in the same position in Figure 3(b) which is obtained from the DNA propeller twist value. The result here shows that DNA structural properties can provide better results than simple numerical indicator sequences for the $1 / 3$ frequency detection.

In this paper, we carry out the conversion based on the structural properties of DNA sequence. The four properties used in the conversion are DNA-bending stiffness [20,21], disrupt energy $[21,22]$, free energy $[21,23]$ and propeller twist $[21,24]$. These four structural properties are selected out of a total of 14 structural properties [21]. In the selection process, firstly the DNA sequences are converted into numerical values based on the 14 structural features, respectively.
The 14 structural features are A philicity, B-DNA twist, bendability, bending stiffness, denaturation, disrupt energy, free energy, GC trinucleotide content, nucleosome positioning, propeller twist, protein DNA twist, protein induced deformability, stacking energy, and Z-DNA stabilizing energy [21]. Then the power spectral density (PSD) of each signal is analyzed. The area under the ROC curve (AUC) is used as the evaluation criterion. A larger AUC value indicates a better performance. We tested on the DNA sequence with NCBI accession number Z20656. We set the AUC threshold to be 0.8 and selected 4 out of 14 structural properties for further analysis. The ROC curves obtained by the 14 structural properties are depicted in Figure 4. The ROC curves obtained by the four selected properties are shown in red. The other curves which are not selected for further computation are in blue.

The physical meanings of the properties are as follows. The bending stiffness is regarded as the string correlation with the anisotropic flexibility of the DNA [20, 21]. The values of bending stiffness are given in $\mathrm{nm}$. The values stand for the persistence length value that is derived from the experimental data [21]. Regions with a high disrupt energy value will be more stable than a region with a lower energy value $[21,22]$. Regions with low free energy content will be more stable than regions with higher free energy content $[21,23]$. The dinucleotide propeller twist is the twist angle measured in degrees $[21,24]$.

\subsection{Moving Window-Based Approach for Nonstationary} Signal Analysis. If we convert a DNA sequence into a digital signal, the signal is nonstationary in nature since different regions of the sequence contain different frequency components. Many traditional signal processing methods including the DFT are based on the premise that the signal is stationary. It is important to use nonstationary signal processing methods to analyze a DNA sequence.

The solution to this problem is that we can deploy a moving window. For each window location, we analyze only the data within the window. The idea behind this approach is that we assume that the signal is stationary within a short piece of sequence though it is not stationary over the entire sequence. The idea is similar to the spectrogram based method widely used in speech signal processing. However, we are only interested in the $1 / 3$ frequency component rather than the full frequency spectrum at each base along the DNA sequence in the exon detection process.

In addition, we analyze multiple input signals at the same time since they all contain the $1 / 3$ frequency component. A moving window is applied to the four signals obtained from the four DNA structural properties. The size of the window will be several times as large as the fundamental repeating unit, which in this case is three.

2.3. Multiscale Spectrum Analysis. According to the Heisenberg Uncertainty Principle, one cannot know what spectral components exist at what instances of times. What one can know is which frequencies exist at what intervals of time. In addition, the better the frequency resolution we have, 


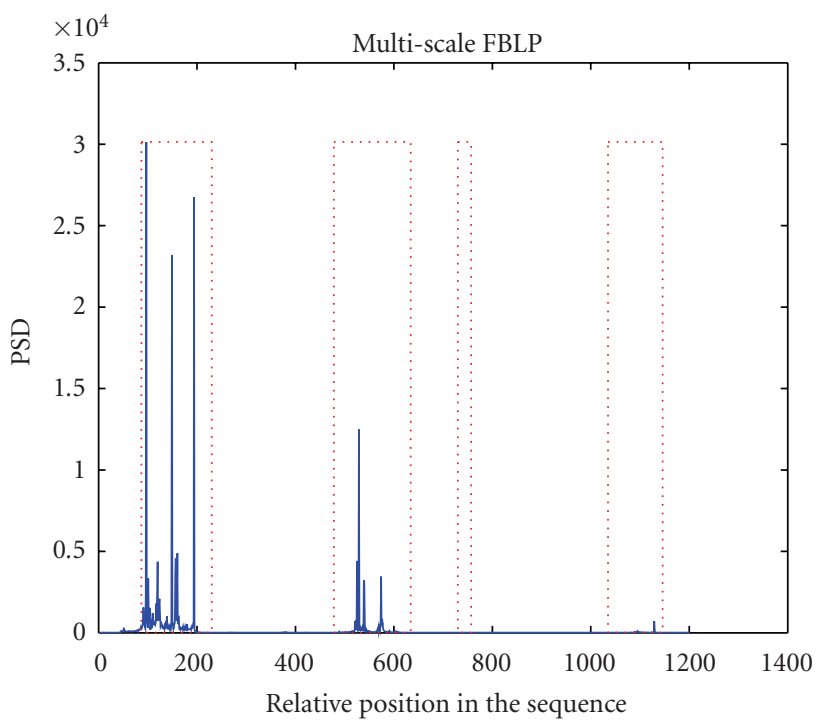

(a)

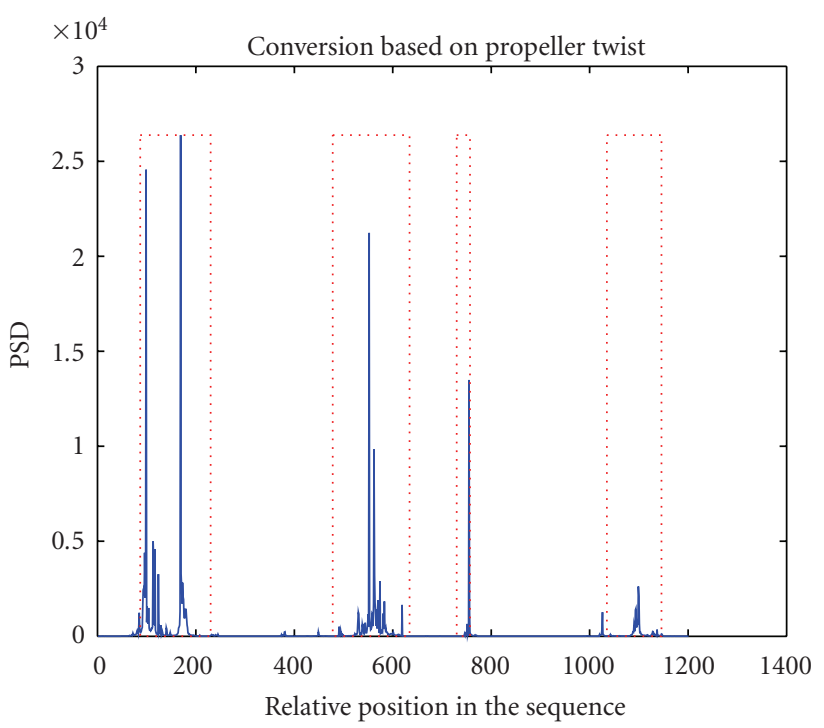

(b)

Figure 3: (a) The PSD obtained from multiscale FBLP method applied to the indicator seqeunces. (b) The PSD obtained by applying the AR modeling method to the DNA propeller twist value.

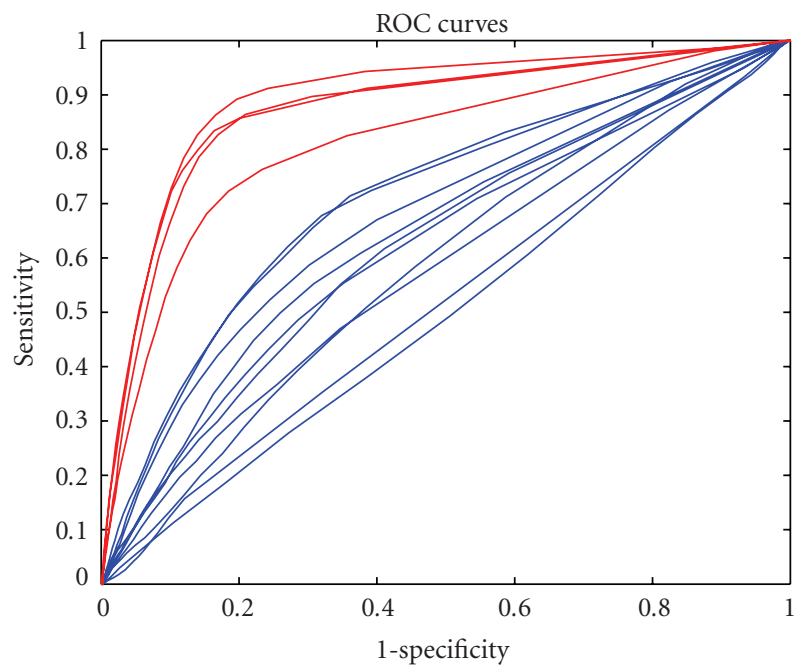

FIGURE 4: ROC curves obtained from the 14 structural properties.

the worse time resolution we get and vice versa. When we apply the principle to our problem, it becomes a tradeoff between frequency resolution and position resolution. In order to know what frequency content is contained in a region, we have to apply a moving window along the sequence. Of course, the better the location information we have, the worse the frequency resolution we get and vice versa. As a result, in order to obtain more accurate information in both frequency and location aspects, we process the signals using several different moving window sizes.
As is already known, different window sizes may produce different spectral estimation results. Large window sizes may miss short exons but produce more accurate results for long exons. Small window sizes may cause more false alarms but will not miss short exons. Multiscale spectrum analysis is equivalent to wavelet analysis [25] in terms of joint frequency and position localization. We use the AR model instead of wavelets here because the AR model can provide more precise information about the $1 / 3$ frequency component for short signals. Also multiscale spectrum analysis is proven to work better than fixed windows in exon detection [16]. The purpose of deploying multiscale is to overcome the drawbacks in using either small or large window sizes and reinforcing their advantages. The window size is chosen to be 30,60, 90 and 120 in our approach.

2.4. AR Model and PSD. An autoregressive (AR) model is a spectral estimation technique. An AR model can overcome short signal problems, give a higher resolution and produce smaller artifacts for spectral estimation compared with the DFT [15]. The details of the AR model are described below.

Let $\mathbf{S}=\left[y_{1}, y_{2}, y_{3}, \ldots, y_{t}, \ldots, y_{n}\right]$ be a stationary time series which follows an AR model of order. The AR model in matrix form can be described as

$$
\mathbf{y}=\mathbf{Y a}+\varepsilon
$$

where $\mathbf{a}$ is the AR model coefficients and $\varepsilon$ is a noise sequence which is assumed to be normally distributed, with zero mean and variance $\sigma^{2}$. 
If we use the forward-backward linear prediction method, (4) can be written as:

$$
\begin{aligned}
& {\left[\begin{array}{c}
y[p+1] \\
y[p+2] \\
\vdots \\
y[n] \\
y[1] \\
y[2] \\
\vdots \\
y[n-p]
\end{array}\right]} \\
& =\left[\begin{array}{cccc}
y[p] & y[p-1] & \cdots & y[1] \\
y[p+1] & y[p] & \cdots & y[2] \\
\vdots & \vdots & & \vdots \\
y[n-1] & y[n-2] & \cdots & y[n-p] \\
y[2] & y[3] & \cdots & y[p+1] \\
y[3] & y[4] & \cdots & y[p+2] \\
\vdots & \vdots & & \vdots \\
y[n-p+1] & y[n-p+2] & \cdots & y[n]
\end{array}\right] \\
& \times\left[\begin{array}{c}
a_{1} \\
a_{2} \\
a_{3} \\
\vdots \\
\vdots \\
\vdots \\
a_{p-1} \\
a_{p}
\end{array}\right]+\varepsilon_{j} .
\end{aligned}
$$

Equation (5) can be ill-conditioned or inconsistent in many applications. In these cases, we can use singular value decomposition (SVD) to overcome the problem. That is, matrix $\mathbf{Y}$ is decomposed into three matrices as follows:

$$
\begin{aligned}
\mathbf{Y}_{p \times[2 \times(n-p)]}= & \mathbf{U}_{p \times[2 \times(n-p)]} \boldsymbol{\Lambda}_{[2 \times(n-p)] \times[2 \times(n-p)]} \\
& \times \mathbf{V}^{\mathrm{T}}[2 \times(n-p)] \times[2 \times(n-p)],
\end{aligned}
$$

where $\Lambda$ is a diagonal matrix containing singular values:

$$
\Lambda_{[2 \times(n-p)] \times[2 \times(n-p)]}=\left[\begin{array}{cccc}
\lambda_{1} & 0 & 0 & 0 \\
0 & \lambda_{2} & 0 & 0 \\
\vdots & \vdots & \ddots & \vdots \\
0 & 0 & 0 & \lambda_{2 \times(n-p)}
\end{array}\right]=\operatorname{diag}\left(\lambda_{j}\right) .
$$

In order to reduce noise effect, we can rank singular values as:

$$
\lambda_{1} \leq \lambda_{2} \leq \cdots \leq \lambda_{2 \times(n-p)} .
$$

Then we replace small $\lambda_{j}$ values with zero.

The AR coefficients can then be found from the following equation:

$$
\mathbf{a}=\mathbf{V}_{[2 \times(n-p)] \times[2 \times(n-p)]} \boldsymbol{\Lambda}_{[2 \times(n-p)] \times[2 \times(n-p)]} \mathbf{U}^{\mathrm{T}}{ }_{p \times[2 \times(n-p)]} \mathbf{y},
$$

where $\Lambda_{[2 \times(n-p)] \times[2 \times(n-p)]}^{-1}=\operatorname{diag}\left(1 / \lambda_{j}\right)$.

The prediction order $p$ is chosen to be $N / 2$ where $N$ refers to window size. The reason for selecting this order is that Lang and McClellan recommended that the number of AR coefficients should be in the range of N/3 and N/2 for the best frequency estimation [26].

In our approach, a modified AR model-based spectral estimation method is used. The idea is that since the four signals are obtained based on the same DNA sequence, their AR coefficients $\mathbf{a}_{1}$ to $\mathbf{a}_{4}$, of the signals should be similar to each other. Hence we can stack the four matrices obtained from each model before doing singular value decomposition. It is expected that a better noise filtering effect will be achieved. The detailed method is described below:

Assume that the AR model for the DNA-bending stiffness, disrupt energy, free energy and propeller twist are, respectively,

$$
\begin{aligned}
& \mathbf{y}_{1}=Y_{1} \mathbf{a}_{1}+\varepsilon, \\
& \mathbf{y}_{2}=Y_{2} \mathbf{a}_{2}+\varepsilon, \\
& \mathbf{y}_{3}=Y_{3} \mathbf{a}_{3}+\varepsilon, \\
& \mathbf{y}_{4}=Y_{4} \mathbf{a}_{4}+\varepsilon .
\end{aligned}
$$

That is, we establish an AR model in (4) and (5) for each of the four structural properties.

Note that the original signals should be normalized to the range of -1 to 1 before constructing the matrices. Then we combine the four matrices together as

$$
\mathrm{Q}=\left[\begin{array}{l}
\mathrm{Y}_{1} \\
\mathrm{Y}_{2} \\
\mathrm{Y}_{3} \\
\mathrm{Y}_{4}
\end{array}\right]
$$

Each of the Matrices $\mathbf{Y}_{1}, \mathbf{Y}_{2}, \mathbf{Y}_{3}, \mathbf{Y}_{4}$ is composed of two individual Toeplitz matrices. However, the combined matrix $\mathbf{Q}$ is not Toeplitz matrix but a block Toeplitz matrix.

We apply singular value decompositions to, compute, rank the singular values and zero the small ones. Then we compute the noise-reduced $\mathbf{Q}$ by

$$
\mathbf{Q}=\mathbf{U} \Lambda \mathbf{V}^{\mathrm{T}},
$$

where $\Lambda$ is a new diagonal matrix containing processed singular values. 
Then we average the values in each descending diagonal in each Toeplitz matrix and put the averaged value back to their original position. After that, we carry out singular value decomposition to $\mathbf{X}$ and compute the AR coefficients according to (6), (7) and (9).

Finally, power spectral density (PSD) can be calculated based on the following equation:

$$
P_{\mathrm{AR}}(\omega)=\frac{\sigma^{2}}{\left|1+\sum_{k=1}^{p} a_{k} \exp (-j \omega k)\right|^{2}},
$$

where $\sigma^{2}$ is the variance of noise.

\section{Experiment Results}

In order to assess the performance of the proposed algorithms, a total of 28 sequences with length between $20000 \mathrm{bp}$ and $40000 \mathrm{bp}$ are downloaded from NCBI GenBank database. There are 564 exons in the sequences. The NCBI accession numbers for these DNA sequences are AB006684, AB022785, AB044947, AB088096, AB088098, AX000035, AX000057, AX259776, AX589170, AX698292, AX814795, AX938514, CQ894214, AB088115, AB103596, $\mathrm{AB} 103602, \mathrm{AB} 103604, \mathrm{AB} 202086, \mathrm{AB} 202093, \mathrm{AB} 202094$, AB202095, AB202112, AF004877, AF026276, AF026801, AF039401, AF178081, Z20656. The total sequence length is 743378 bp.

We have compared our exon detection results with those from the discrete wavelet transform denoise method [18] and the multiscale FBLP method [16] as well as a simple PSD addition method. Two evaluation criteria are used in the comparison. The first one is the Receiver Operating Characteristic (ROC) curve and the area under the ROC curve (AUC). This criterion is used to evaluate the sensitivity and specificity of each method and its overall performance. The second evaluation criterion is the rate of correct detection of short exons, each of which is no longer than $70 \mathrm{bp}$.

In the simple PSD addition method, we compute the PSD for each of the four DNA structural signals. Then the four PSDs are added to obtain one PSD which is used for the ROC curve analysis as well as short exon detection.

To draw the ROC curve, we shall firstly quantize the PSD values. Then set the threshold value to be the smallest value of the quantized PSD. All the values greater than the threshold value are considered to be the indication of exonic areas while all the values lower than the threshold values are considered to be the indication of intronic areas. Then we compute true negative, false negative, true positive and false positive values. After that, the specificity and sensitivity values are computed as in

$$
\begin{aligned}
& \text { Specificity }=\frac{\text { True Negatives }}{\text { True Negatives + False Positives }}, \\
& \text { Sensitivity }=\frac{\text { True Positives }}{\text { True Positives + False Negatives }} .
\end{aligned}
$$

Each time we will set the threshold value to be one which is larger than the current one value to obtain new
TABle 1: Area under the ROC curve (AUC) for human DNA sequences.

\begin{tabular}{ccccc}
\hline $\begin{array}{c}\text { Multiscale } \\
\text { FBLP }\end{array}$ & $\begin{array}{c}\text { DWT } \\
\text { de-noise }\end{array}$ & Simple addition & Multifeature \\
\hline AUC & 0.63 & 0.68 & $\mathbf{0 . 7 2}$ & $\mathbf{0 . 7 2}$ \\
\hline
\end{tabular}

TABLe 2: Sensitivity and specificity at optimal cutoff point for human DNA sequences.

\begin{tabular}{lcccc}
\hline & $\begin{array}{c}\text { Multiscale } \\
\text { FBLP }\end{array}$ & $\begin{array}{c}\text { DWT } \\
\text { de-noise }\end{array}$ & Simple addition & Multifeature \\
\hline Sensitivity & 0.32 & 0.42 & 0.57 & $\mathbf{0 . 5 9}$ \\
Specificity & $\mathbf{0 . 9 4}$ & 0.89 & 0.78 & 0.76 \\
\hline
\end{tabular}

sensitivity and specificity values until we reach the largest quantized value. Finally, we draw ROC curves based on all the specificity and sensitivity values. It shall be pointed out that we take logarithm of the PSD to amplify the signal before quantization for the multiscale FBLP, simple addition and multifeature spectral analysis methods.

The ROC curves for the four algorithms are shown in Figure 5 and the AUC values are given in Table 1. Improvement of the results is noticed as the AUC of our method is larger than the other three methods. In Figure 5, although the ROC curve obtained by multiscale FBLP method is higher than that of our method in the interval $[0,0.12]$, our method has an overall much better performance.

The optimal cutoff point is decided based on the Youden's index [27]. The sensitivity and specificity values are given in Table 2. From Table 2, we observe that our method has the highest sensitivity value while multiscale FBLP method has the highest specificity value. Our method increases the sensitivity by 0.27 with a 0.18 decrease of specificity compared with the multiscale FBLP method and increases the sensitivity by 0.17 with a 0.11 decrease of specificity compared with the DWT denoise method. For the same sensitivity, our method produces the best specificity. And for the same specificity, our method produces the best sensitivity. That is, overall our method performs the best as it produces the largest area under the ROC.

The performances of short exon detection methods are presented in Table 3. The short exon positions are identified first. Then every nucleotide within each short exon is labeled positive or negative according to the optimal cutoff point value obtained from previous steps. If the number of nucleotides which are labeled positive composes $80 \%$ or more of the exon region, the exon is considered being detected. From Table 3, it is observed that our method for short exon detection is superior to the other two methods.

We should also point out here that the detection results of multifeature spectral analysis are not a simple combination of the detection results from four features analyzed separately. From Table 3, it can be seen that the detection results of multifeature spectral analysis surpasses that of the simple addition method by $10.4 \%$. The experiment results demonstrate the effectiveness of our multifeature based approach. 


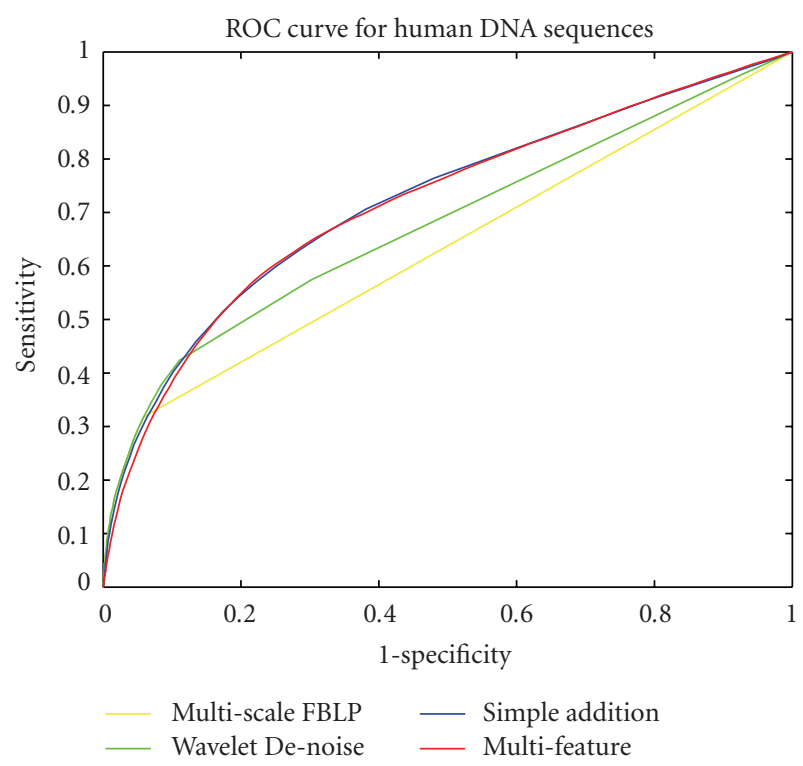

Figure 5: ROC curves obtained by four methods for human DNA sequences.

TABLE 3: Short exon detection results for human DNA sequences.

\begin{tabular}{lcccc}
\hline & $\begin{array}{c}\text { Multiscale } \\
\text { FBLP }\end{array}$ & $\begin{array}{c}\text { DWT } \\
\text { de-noise }\end{array}$ & Simple addition & Multifeature \\
\hline $\begin{array}{l}\text { Number of } \\
\text { exons } \\
\text { detected }\end{array}$ & $9 / 135$ & $0 / 135$ & $44 / 135$ & $\mathbf{6 0 / 1 3 5}$ \\
$\begin{array}{l}\text { Detection } \\
\text { success rate }\end{array}$ & $6.7 \%$ & $0.0 \%$ & $32.6 \%$ & $\mathbf{4 4 . 4} \%$ \\
\hline
\end{tabular}

TAble 4: Area under the ROC curve (AUC) for mouse DNA sequences.

\begin{tabular}{ccccc}
\hline & $\begin{array}{c}\text { Multiscale } \\
\text { FBLP }\end{array}$ & $\begin{array}{c}\text { DWT } \\
\text { de-noise }\end{array}$ & Simple addition & Multifeature \\
\hline AUC & 0.62 & 0.63 & 0.65 & $\mathbf{0 . 6 6}$ \\
\hline
\end{tabular}

We also tested our method on 7 short mouse DNA sequences with NCBI accession numbers AB025024, AB040292, AB052362, AF040759, AF068865, AF203031, and AJ298076. The total length of the 7 Mouse sequence is $175298 \mathrm{bp}$. There are 112 exons among which 13 exons are no longer than $70 \mathrm{bp}$. From Table 5, we can see that at the optimal cutoff point, our method can obtain the largest sensitivity value while multiscale FBLP can obtain the largest specificity value. From Figure 6, it is observed that for the same sensitivity value, our method obtains the best specificity value. For the same specificity value, our method produces the best sensitivity value. Our method produces the largest AUC value as shown in Table 4 and has the best overall performance.

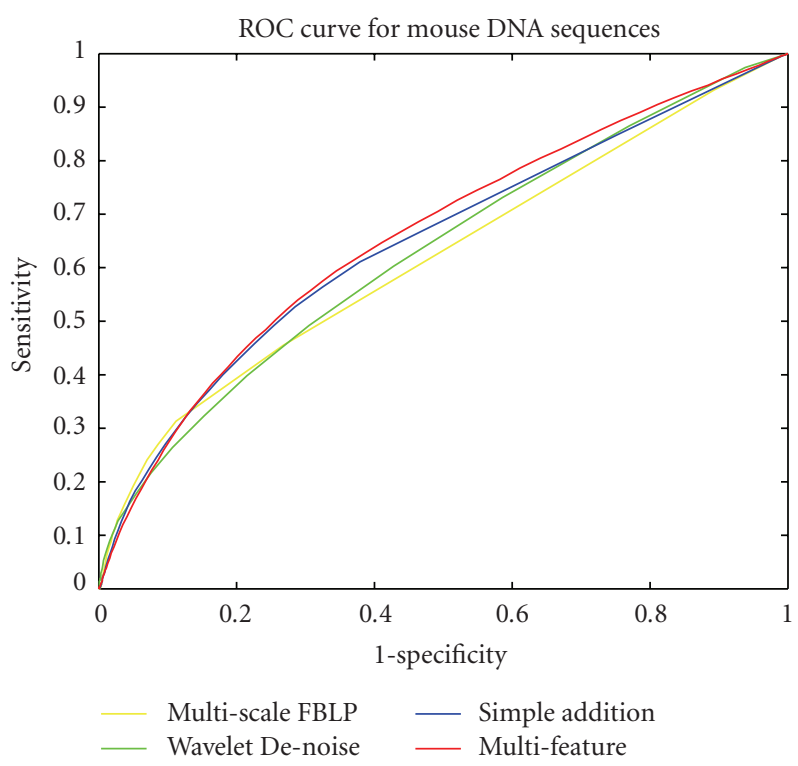

Figure 6: ROC curves obtained by four methods for mouse DNA sequences.

TABle 5: Sensitivity and specificity at optimal cutoff point for mouse DNA sequences.

\begin{tabular}{lcccc}
\hline & $\begin{array}{c}\text { Multiscale } \\
\text { FBLP }\end{array}$ & $\begin{array}{c}\text { DWT } \\
\text { de-noise }\end{array}$ & Simple addition & Multifeature \\
\hline Sensitivity & 0.31 & 0.49 & 0.53 & $\mathbf{0 . 5 4}$ \\
Specificity & $\mathbf{0 . 8 9}$ & 0.70 & 0.71 & 0.71 \\
\hline
\end{tabular}

TABLE 6: Short exon detection results for mouse DNA sequences.

\begin{tabular}{lcccc}
\hline & $\begin{array}{c}\text { Multiscale } \\
\text { FBLP }\end{array}$ & $\begin{array}{c}\text { DWT } \\
\text { de-noise }\end{array}$ & Simple addition & Multifeature \\
\hline $\begin{array}{l}\text { Number of } \\
\text { exons } \\
\text { detected }\end{array}$ & $2 / 13$ & $0 / 13$ & $2 / 13$ & $\mathbf{4} 13$ \\
$\begin{array}{l}\text { Detection } \\
\text { success rate }\end{array}$ & $15.4 \%$ & $0.0 \%$ & $15.4 \%$ & $\mathbf{3 0 . 8} \%$ \\
\hline
\end{tabular}

\section{Conclusion}

Short exon detection is difficult because the spectral component of period three is very weak in the exon regions. In this paper, we have proposed a multifeature spectral analysis method to solve this problem. Four discrete signals are obtained from a DNA sequence based on four structural properties, the DNA-bending stiffness, disrupt energy, free energy and propeller twist. All these signals contain the $1 / 3$ frequency component. We apply the AR model-based spectral analysis to the four signals by combining their linear prediction matrices and performing SVD-based filtering to reduce noise. Moving windows with different sizes are used to overcome the nonstationarity of DNA sequences. The exon detection results from multifeatures are better than the combination of the detection results from the four features separately. In addition, we have compared the results from 
the proposed method with those obtained from multiscale FBLP [16] and discrete wavelet transform denoise [18] methods. Experiment results show that our method is superior in short exon detection to the existing signal processing-based techniques. Further increase in detection accuracy is possible if we combine the proposed method with supervised machine learning algorithms and string matching based techniques.

\section{Acknowledgment}

This work is supported by a Grant from the Hong Kong Research Grant Council (Project CityU 123809).

\section{References}

[1] J. D. Watson, T. A. Baker, S. P. Bell et al., "RNA splicing," in Molecular Biology of the Gene, chapter 13, Cold Spring Harbor Laboratory Press, Cold Spring Harbor, NY, USA, 6th edition, 2008.

[2] C. Mathé, M.-F. Sagot, T. Schiex, and P. Rouzé, "Current methods of gene prediction, their strengths and weaknesses," Nucleic Acids Research, vol. 30, no. 19, pp. 4103-4117, 2002.

[3] J. D. Hawkins, "A survey on intron and exon lengths," Nucleic Acids Research, vol. 16, no. 21, pp. 9893-9908, 1988.

[4] J. W. Fickett, "Recognition of protein coding regions in DNA sequences," Nucleic Acids Research, vol. 10, no. 17, pp. 53035318, 1982.

[5] X. Xie, S. Wu, K.-M. Lam, and H. Yan, "PromoterExplorer: an effective promoter identification method based on the AdaBoost algorithm," Bioinformatics, vol. 22, no. 22, pp. 27222728, 2006.

[6] S. Wu, X. Xie, A. W.-C. Liew, and H. Yan, "Eukaryotic promoter prediction based on relative entropy and positional information," Physical Review E, vol. 75, no. 4, Article ID 041908, 7 pages, 2007.

[7] R. Durbin, S. Eddy, A. Krogh, and G. Mitchison, Biological Sequence Analysis: Probabilistic Models of Proteins and Nucleic Acids, Cambridge University Press, Cambridge, UK, 1998.

[8] J. V. Lorenzo-Ginori, A. Rodríguez-Fuentes, R. G. Ábalo, and R. S. Rodríguez, "Digital signal processing in the analysis of genomic sequences," Current Bioinformatics, vol. 4, no. 1, pp. 28-40, 2009.

[9] S. Tiwari, S. Ramachandran, A. Bhattacharya, S. Bhattacharya, and R. Ramaswamy, "Prediction of probable genes by Fourier analysis of genomic sequences," Computer Applications in the Biosciences, vol. 13, no. 3, pp. 263-270, 1997.

[10] M. Akhtar, E. Ambikairajah, and J. Epps, "Optimizing period3 methods for eukaryotic gene prediction," in Proceedings of the IEEE International Conference on Acoustics, Speech and Signal Processing (ICASSP '08), pp. 621-624, 2008.

[11] P. P. Vaidyanathan and B.-J. Yoon, "Gene and exon prediction using allpass-based filters," in Proceedings of the IEEE International Workshop on Genomic Signal Processing and Statistics (GENSIPS '02), Raleigh, NC, USA, October 2002.

[12] R. Román-Roldán, P. Bernaola-Galván, and J. L. Oliver, "Sequence compositional complexity of DNA through an entropic segmentation method," Physical Review Letters, vol. 80, no. 6, pp. 1344-1347, 1998.

[13] D. Nicorici and J. Astola, "Segmentation of DNA into coding and noncoding regions based on recursive entropic segmentation and stop-codon statistics," EURASIP Journal on Applied Signal Processing, vol. 2004, no. 1, pp. 81-91, 2004.
[14] N. Chakravarthy, A. Spanias, L. D. Iasemidis, and K. Tsakalis, "Autoregressive modeling and feature analysis of DNA sequences," EURASIP Journal on Applied Signal Processing, vol. 2004, no. 1, pp. 13-28, 2004.

[15] H. Yan and T. D. Pham, "Spectral estimation techniques for DNA sequence and microarray data analysis," Current Bioinformatics, vol. 2, no. 2, pp. 145-156, 2007.

[16] M. K. Choong and H. Yan, "Multi-scale parametric spectral analysis for exon detection in DNA sequences based on forward-backward linear prediction and singular value decomposition of the double-base curves," Bioinformation, vol. 2, no. 7, pp. 273-278, 2008.

[17] R. Jiang and H. Yan, "Studies of spectral properties of short genes using the wavelet subspace Hilbert-Huang transform (WSHHT)," Physica A, vol. 387, no. 16-17, pp. 4223-4247, 2008.

[18] T. P. George and T. Thomas, "Discrete wavelet transform denoising in eukaryotic gene splicing," BMC Bioinformatics, vol. 11 , supplement 1 , article S50, 2010.

[19] Y. Wu, A. W.-C. Liew, H. Yan, and M. Yang, "DB-Curve: a novel 2D method of DNA sequence visualization and representation," Chemical Physics Letters, vol. 367, no. 1-2, pp. 170-176, 2003.

[20] A. V. Sivolob and S. N. Khrapunov, "Translational positioning of nucleosomes on DNA: the role of sequence-dependent isotropic DNA bending stiffness," Journal of Molecular Biology, vol. 247, no. 5, pp. 918-931, 1995.

[21] K. Florquin, Y. Saeys, S. Degroeve, P. Rouzé, and Y. Van de Peer, "Large-scale structural analysis of the core promoter in mammalian and plant genomes," Nucleic Acids Research, vol. 33, no. 13, pp. 4255-4264, 2005.

[22] K. J. Breslauer, R. Frank, H. Blocker, and L. A. Marky, "Predicting DNA duplex stability from the base sequence," Proceedings of the National Academy of Sciences of the United States of America, vol. 83, no. 11, pp. 3746-3750, 1986.

[23] N. Sugimoto, S.-I. Nakano, M. Yoneyama, and K.-I. Honda, "Improved thermodynamic parameters and helix initiation factor to predict stability of DNA duplexes," Nucleic Acids Research, vol. 24, no. 22, pp. 4501-4505, 1996.

[24] M. A. El Hassan and C. R. Calladine, "Propeller-twisting of base-pairs and the conformational mobility of dinucleotide steps in DNA," Journal of Molecular Biology, vol. 259, no. 1, pp. 95-103, 1996.

[25] P. Yiou, D. Sornette, and M. Ghil, "Data-adaptive wavelets and multi-scale singular-spectrum analysis," Physica D, vol. 142, no. 3-4, pp. 254-290, 2000.

[26] S. W. Lang and J. H. McClellan, "Frequency estimation with maximum entropy spectral estimator," IEEE Transactions on Acoustics, Speech, and Signal Processing, vol. 28, no. 6, pp. 716$724,1980$.

[27] W. J. Youden, "Index for rating diagnostic tests," Cancer, vol. 3, no. 1, pp. 32-35, 1950. 